\title{
Indocyanine green assembled free oxygen-nanobubbles towards enhanced near-infrared induced photodynamic therapy
}

\author{
Li Yang ${ }^{1}$, Bin Huang ${ }^{1,2}(\bowtie)$, Shiqi Hu${ }^{3}$, Yuan $\mathrm{An}^{1}$, Jingyi Sheng ${ }^{1}$, Yan $\mathrm{Li}^{1}$, Yuxin Wang ${ }^{3}$, and Ning Gu ${ }^{1}(\bowtie)$ \\ ${ }^{1}$ State Key Laboratory of Bioelectronics, Jiangsu Key Laboratory for Biomaterials and Devices, School of Biological Science and Medical Engineering, \\ Southeast University, Nanjing 210096, China \\ ${ }^{2}$ College of Life Sciences and Chemistry, Jiangsu Second Normal University, Nanjing 210013, China \\ ${ }^{3}$ Nanjing Stomatology Hospital, Nanjing 210008, China
}

(c) Tsinghua University Press 2022

Received: 16 October 2021 / Revised: 15 December 2021 / Accepted: 19 December 2021

\begin{abstract}
Photodynamic therapy (PDT) has shown a promising capability for cancer treatment with minimal side effects. Indocyanine green (ICG), the only clinically approved near-infrared (NIR) fluorophore, has been used as a photosensitizer for PDT in clinical application. However, the main obstacle of directly utilizing ICG in the clinic lies in its low singlet oxygen $\left({ }^{1} \mathrm{O}_{2}\right)$ quantum yield $(\mathrm{QY})$ and instability in aqueous solution. To improve the PDT efficacy of ICG, free ICG molecules were assembled with free oxygen nanobubbles $\left(\mathrm{NBs}-\mathrm{O}_{2}\right)$ to fabricate ICG-NBs- $\mathrm{O}_{2}$ by hydrophilic-hydrophobe interactions on the gas-liquid interface. Interestingly, ${ }^{1} \mathrm{O}_{2}$ QY of ICG-NBs-O ${ }_{2}$ solution was significantly increased to $1.6 \%$, which was estimated to be 8 times as high as that of free ICG solution. Meanwhile, ICG-NBs- $\mathrm{O}_{2}$ exhibited better aqueous solution stability compared with free ICG. Furthermore, through establishing tumor models in nude mice, the therapeutic efficacy of ICG-NBs- $\mathrm{O}_{2}$ was also assessed in the PDT treatment of oral cancer. The tumor volume in ICG-NBs- $\mathrm{O}_{2}$ treated group on day 14 decreased to 0.56 of the initial tumor size on day 1 , while the tumor volume in free ICG treated group increased to 2.4 times. The results demonstrated that ICG-NBs- $\mathrm{O}_{2}$ showed excellent tumor ablation in vivo. Therefore, this facile method provided an effective strategy for enhanced PDT treatment of ICG and showed great potential in clinical application.
\end{abstract}

\section{KEYWORDS}

free oxygen-nanobubbles, aqueous solution stability, singlet oxygen quantum yield, photodynamic therapy, indocyanine green

\section{Introduction}

In comparison with traditional cancer therapies, phototherapy has attracted increasing attention in recent years due to its selective and localized therapeutic effect with minimal invasion for tumor treatment [1-5]. Particularly, photodynamic therapy (PDT) has shown capable potential with high specificity and low side effects towards clinical applications [6-11]. In principle, PDT provides potential for killing tumor cells with reactive oxygen species (ROS) or singlet oxygen $\left({ }^{1} \mathrm{O}_{2}\right)$, which are generated by the combination of three factors such as appropriate optical irradiation, photosensitizer molecules, and environmental oxygen [12-18]. To achieve efficient PDT, considerable attention has been paid to design and synthesize new photosensitizers [19-22] with efficient ROS or ${ }^{1} \mathrm{O}_{2}$ efficiency. However, for a new photosensitizer, a key issue that must be addressed is the careful evaluation in vitro and in vivo for safety. As a result, the practical biological applications of most newly synthesized photosensitizers are still in their infancy. On the other hand, the tumor hypoxia with insufficient oxygen supply during PDT treatment may result in low ${ }^{1} \mathrm{O}_{2}$ production and decreased PDT efficacy [23-25]. Therefore, the increase of oxygen supply may provide another strategy to enhance the production of ${ }^{1} \mathrm{O}_{2}$ for efficient PDT. Some oxidants such as $\mathrm{MnO}_{2}$, $\mathrm{H}_{2} \mathrm{O}_{2}, \mathrm{Fe}^{3+}$, etc., were developed to increase the oxygen supply for enhanced PDT [26]. However, the addition of oxidants may change the tumor microenvironment and induce antioxidant response [27], which may impair PDT efficiency. Up to now, the PDT agents which can cope with these disadvantages and be really suitable for clinical utilization are still inadequate. It is urgent to develop a convenient method to increase the oxygen supply for efficient PDT.

Indocyanine green (ICG) is the only near-infrared (NIR) photosensitizer approved by the U.S. Food and Drug Administration (FDA) [28-31]. Under NIR laser irradiation, ICG can effectively produce ROS or ${ }^{1} \mathrm{O}_{2}$ for PDT in the tumor environment [32]. However, the application of ICG is hindered by several intrinsic limitations, including concentration-dependent aggregation and poor aqueous stability. Simultaneously, the therapeutic effect of free ICG as a PDT agent is also restricted to the hypoxia tumor environment. To overcome these limitations, various ICG-based nanoparticles have been developed. For instance, $\mathrm{Wu}$ et al. developed a polymeric micelle encapsulated with ICG to improve the aqueous stability of ICG [33]. Lee et al. reported that ICG molecules encapsulated inside the nanochannels of mesoporous silica nanoparticles might prevent them from aggregation [34]. Patel et al. revealed that the optical stability can be improved by entrapping ICG with polymer 
nanocomposites [35]. However, during the complicated synthesis process, the introduction of new compositions other than ICG molecules may increase the toxicity of ICG nanostructures. Therefore, it is an important issue to develop ICG based agents with simple ingredients which can fulfill their full potential in PDT.

To improve the PDT efficacy of ICG, some factors such as the increase of oxygen supply and aqueous stability need to be considered. According to our recent work [36], the aqueous stability of ICG can be enhanced by assembling with $\mathrm{SF}_{6}$ nanobubbles (NBs). Therefore, the combination of free oxygen NBs and aqueous ICG may provide an effective strategy to meet the above-mentioned requirements. In this study, free ICG solution was self-assembled with free oxygen-NBs $\left(\mathrm{NBs}-\mathrm{O}_{2}\right)$ to fabricate ICG-NBs- $\mathrm{O}_{2}$ solution. The hydrodynamic diameter of ICG-NBs- $\mathrm{O}_{2}$ sample was characterized by dynamic light scattering (DLS) measurement, and morphology was visualized via transmission electron microscopy (TEM). In addition, the aqueous stability of ICG-NBs- $\mathrm{O}_{2}$ was monitored with ultraviolet-visible-NIR (UV-Vis-NIR) spectrophotometer. The results indicated that the aqueous stability of ICG-NBs- $\mathrm{O}_{2}$ solution was significantly improved compared with free ICG solution. Furthermore, the ICG-NBs- $\mathrm{O}_{2}$ solution that served as a photosensitizer for PDT was also investigated. Interestingly, the ${ }^{1} \mathrm{O}_{2}$ quantum yield (QY) of ICG-NBs- $\mathrm{O}_{2}$ solution was significantly increased to $1.6 \%$, which is estimated to be 8 times as high as that of free ICG solution. By establishing tumor models in nude mice, the therapeutic efficacy of the ICG-NBs- $\mathrm{O}_{2}$ was also assessed in the PDT treatment of oral cancer. The results demonstrated that ICG-NBs- $\mathrm{O}_{2}$ exhibited outstanding treatment effect in tumor ablation in vivo under NIR laser irradiation. Therefore, this work provided a simple and facile approach to enhance the therapeutic effect of ICG in PDT treatments.

\section{Materials and methods}

\subsection{Materials}

Ultrapure water with a resistivity of $18.25 \mathrm{M} \Omega \cdot \mathrm{cm}$ was processed in a water purification system (Youpu Ultrapure Technology Co., Ltd., China). Oxygen with a purity of $99.6 \%$ was purchased from Zhende Medical Co., Ltd (Shaoxing, China). $\mathrm{SF}_{6}$ and Xe with a purity of 99.99\% were purchased from Anhui Qiangyuan Gas Co., Ltd. (Wuhu, China). $\mathrm{N}_{2}$ with a purity of $99.99 \%$ was obtained from Nanjing Wenda Special Gas Co. Ltd. ICG for injection was acquired from Dandong Yichuang Pharmaceutical Co., Ltd. (Dandong, China). 1,3-Diphenylisobenzofuran (DPBF) was obtained from Aladdin Reagent (Shanghai, China) and dimethyl sulfoxide (DMSO) was supplied by Solarbio Life Science Co., Ltd. (Beijing, China). 4,6-Diamidino-2-phenylindole (DAPI) was purchased from YEASEN (China). Dulbecco's modified Eagle's medium (DMEM, high glucose), penicillin-streptomycin, trypsinEDTA, fetal bovine serum (FBS), and phosphate-buffered saline (PBS, pH 7.4) were obtained from Thermo Fisher Scientific (USA). Cell Counting Kit-8 (CCK-8) and 2,7dichlorodihydrofluorescein diacetate (DCFH-DA) were received from Nanjing KeyGEN Biotech (Nanjing, China).

\subsection{Preparation of the ICG-NBs}

The ICG-NBs- $\mathrm{O}_{2}$ was fabricated according to the self-assembly method reported in our previous work [36]. The free $\mathrm{NBs}-\mathrm{O}_{2}$ solution was prepared with repeated compression method [37]. Firstly, the vial bottle was filled with $2 \mathrm{~mL}$ of ultrapure water and oversaturated $\mathrm{O}_{2}$. Then, a medical syringe filled with $3 \mathrm{~mL} \mathrm{O}$ gas was connected to the vial bottle. So, the syringe and the vial bottle formed an airtight system and the pressure inside the bottle can be changed by moving the syringe piston up and down in the vertical

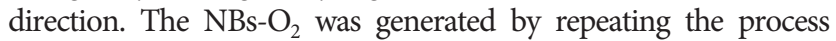
periodically. After that, ICG was dissolved in ultrapure water with concentration of $1 \mathrm{mg} / \mathrm{mL}$. This free ICG solution was added into free NBs- $\mathrm{O}_{2}$ aqueous solution with the final ICG concentration of $10 \mu \mathrm{g} / \mathrm{mL}$. Subsequently, the ICG-NBs- $\mathrm{O}_{2}$ solution was generated via self-assembly. With the same method, replacing $\mathrm{O}_{2}$ with $\mathrm{SF}_{6}$, $\mathrm{N}_{2}$, and $\mathrm{Xe}$, the corresponding ICG-NBs-SF , ICG-NBs- $\mathrm{N}_{2}$, and ICG-NBs-Xe solution were prepared, respectively.

\subsection{Characterization of ICG-NBs-O}

The hydrodynamic diameters and zeta potential of free $\mathrm{NBs}-\mathrm{O}_{2}$ and ICG-NBs- $\mathrm{O}_{2}$ solution were measured by DLS on a Zetasizer Nano ZS90 (Malvern, UK) at room temperature. The refractive index of the material and dispersant were set to 1.0 and 1.33, respectively. The morphology of ICG-NBs- $\mathrm{O}_{2}$ was characterized with TEM (JEM-2100, JEOL Ltd., Japan). The UV-Vis-NIR absorbance spectra were recorded using a spectrophotometer (UV3600, Shimadzu, Japan). The oxygen contents in ultrapure water, free NBs- $\mathrm{O}_{2}$, and ICG-NBs- $\mathrm{O}_{2}$ solution were measured with the Benchtop Dissolved Oxygen Meter (JPSJ-605F, Inesa Scientific Instrument Co., Ltd., China).

\subsection{Photothermal and photodynamic properties of ICG-} NBs- $\mathrm{O}_{2}$

To activate the photothermal and photodynamic effects of ICG and ICG-NBs-O 2 , a NIR laser (wavelength: $808 \mathrm{~nm}$ and power density: $1 \mathrm{~W} / \mathrm{cm}^{2}$ ) was used in the experiment. In characterization of photothermal effects, $2 \mathrm{~mL}$ of ICG and ICG-NBs- $\mathrm{O}_{2}$ solution with the same ICG concentration of $10 \mu \mathrm{g} / \mathrm{mL}$ were transferred into transparent quartz vial and irradiated by NIR laser for $9 \mathrm{~min}$, respectively. The temperature changes during the irradiation were recorded for every $30 \mathrm{~s}$ using an infrared thermal imaging camera (TESTO 869, Germany).

The amount of ${ }^{1} \mathrm{O}_{2}$ generated in a reaction system by ICG and ICG-NBs- $\mathrm{O}_{2}$ solution was evaluated using DPBF as a ${ }^{1} \mathrm{O}_{2}$ sensor. Firstly, $3 \mathrm{~mL}$ of ultrapure water, free ICG solution (ICG concentration: $10 \mu \mathrm{g} / \mathrm{mL}$ ), and ICG-NBs-O ${ }_{2}$ solution (ICG concentration: $10 \mu \mathrm{g} / \mathrm{mL}$ ) each containing $100 \mu \mathrm{L}$ of DPBF solution $(0.5 \mathrm{mg} / \mathrm{mL}$, freshly dissolved in DMSO) were placed in transparent quartz vial. Then each mixture was irradiated by an $808 \mathrm{~nm}$ NIR laser $\left(1 \mathrm{~W} / \mathrm{cm}^{2}\right)$ for $0,2,4,6,8,10$, and $15 \mathrm{~min}$. After the irradiation, the absorbance of DPBF solution was assessed using LAMBDA 365 UV-Vis-NIR Spectrophotometer (PerkinElmer, USA) at $420 \mathrm{~nm}$.

\section{$2.5{ }^{1} \mathrm{O}_{2} \mathrm{QY}$ measurements}

Production of ${ }^{1} \mathrm{O}_{2}$ was determined by time-dependent losses of DPBF absorption. The ${ }^{1} \mathrm{O}_{2}$ QY of ICG-NBs- $\mathrm{O}_{2}$ solution was investigated with an indirect method [38]. Free ICG solution was used as a comparison standard with a ${ }^{1} \mathrm{O}_{2}$ QY of $0.2 \%$ [39]. The ${ }^{1} \mathrm{O}_{2}$ QY of the ICG-NBs- $\mathrm{O}_{2}$ solution was calculated using the following Eq. (1)

$$
\Phi_{\text {sample }}=\Phi_{\text {ICG }}\left(\frac{K_{\text {sample }}}{K_{\text {ICG }}}\right)\left(\frac{A_{\text {ICG }}}{A_{\text {sample }}}\right)
$$

where $K_{\text {sample }}$ and $K_{\mathrm{ICG}}$ represent the decomposition rate constants of DPBF by ICG-NBs-O ${ }_{2}$ and free ICG solution, respectively. $A_{\text {ICG }}$ and $A_{\text {sample }}$ are the integral areas of the optical absorption bands between 750 and $850 \mathrm{~nm}$ by ICG-NBs- $\mathrm{O}_{2}$ and free ICG solution, respectively. 


\subsection{Cell culture}

The human tongue cancer cells Cal27 were grown in high-glucose DMEM supplemented with $10 \%(\mathrm{v} / \mathrm{v})$ FBS and $1 \%(\mathrm{v} / \mathrm{v})$ penicillin/streptomycin. All cells were cultured in a humidified standard incubator containing $5 \% \mathrm{CO}_{2}$ at $37^{\circ} \mathrm{C}$.

\subsection{In vitro cellular uptake}

Cal27 cells were seeded in confocal laser scanning microscopy (CLSM) dishes at a density of $8.0 \times 10^{4}$ cells per dish and cultured for $24 \mathrm{~h}$ in standard incubator $\left(5 \% \mathrm{CO}_{2}, 37^{\circ} \mathrm{C}\right)$. The cells were washed with PBS for three times and then treated with appropriate amount of ICG or ICG-NBs- $\mathrm{O}_{2}$ solution with the same concentration of ICG $(10 \mu \mathrm{g} / \mathrm{mL})$. After incubated further for $12 \mathrm{~h}$, the cells were washed three times with PBS, fixed with $4 \%$ paraformaldehyde $(1 \mathrm{~mL})$ for $20 \mathrm{~min}$ and successively stained with DAPI $(100 \mu \mathrm{L})$ for $15 \mathrm{~min}$. Finally, cells were washed three times and visualized immediately by CLSM (Nikon, Japan).

\subsection{Intracellular ROS detection}

DCFH-DA probe was used to evaluate the generation of intracellular ${ }^{1} \mathrm{O}_{2}$ resulting from ICG-NBs- $\mathrm{O}_{2}$ solution uptake by Cal27 cells after NIR laser irradiation. Cal27 cells were seeded in CLSM dishes at a density of $8.0 \times 10^{4}$ cells per dish for $24 \mathrm{~h}$ and subsequently incubated with ICG-NBs- $\mathrm{O}_{2}$ or free ICG solution for overnight. After removing the medium and washed with PBS, the cells successively were incubated with DCFH-DA $(10 \mu \mathrm{M}, 1 \mathrm{~mL})$ for $20 \mathrm{~min}$. Then the cells were exposed to $808 \mathrm{~nm}$ laser $\left(1 \mathrm{~W} / \mathrm{cm}^{2}\right)$ for $5 \mathrm{~min}$ at the room temperature. Fluorescence images were acquired using a CLSM at once (DCFH-DA, 488/525 nm; ICG, 633/700 nm).

\subsection{In vitro cytotoxicity}

The cell cytotoxicity of the ICG-NBs- $\mathrm{O}_{2}$ was evaluated using a CCK- 8 cell viability assay. Firstly, the Cal27 cells were seeded in a 96-well plate at a density of $8 \times 10^{3}$ per well and cultured in standard cell incubator at $37{ }^{\circ} \mathrm{C}$ for $24 \mathrm{~h}$. Then the cells were washed with $\mathrm{PBS}$ for three times and then treated with a series of ICG-NBs-O ${ }_{2}$ solution (ICG concentration: $2.5,5$, and $10 \mu \mathrm{g} / \mathrm{mL}$ ). After incubated further for $12 \mathrm{~h}$, cells were washed three times with PBS, and the cell metabolic viability was evaluated using a CCK-8 assay.

For phototoxicity, the Cal27 cells were seeded in a 96-well plate at a density of $8 \times 10^{3}$ per well and cultured for $24 \mathrm{~h}$. The culture medium was firstly discarded then replaced with new medium containing gradient concentrations of ICG-NBs- $\mathrm{O}_{2}$ (ICG concentration: $2.5,5$, and $10 \mu \mathrm{g} / \mathrm{mL}$ ) solution to culture for overnight. The cells were irradiated with $808 \mathrm{~nm}$ laser $\left(1 \mathrm{~W} / \mathrm{cm}^{2}\right)$ for $5 \mathrm{~min}$ at the ambient temperature of $4{ }^{\circ} \mathrm{C}$. After incubated another $24 \mathrm{~h}$, cells were washed with PBS for three times and the cell phototoxicity was evaluated using a CCK-8 assay.

\subsection{Animals and tumor models}

Male Balb/c nude mice (4-6 weeks of age) were obtained from Ziyuan Biotechnology Co., Ltd. (Hangzhou, China). Animals received care in accordance with the National Guide for Care and Use of Laboratory Animals and approved by the Institutional Animal Care and Use Committee of Southeast University. To construct the human tongue tumor model, $100 \mu \mathrm{L}$ of Cal27 cells $\left(2 \times 10^{7}\right.$ cells $\left./ \mathrm{mL}\right)$ suspension was injected hypodermically into the flank region. Afterwards, the tumor volume was monitored and calculated as (tumor length) $\times(\text { tumor width })^{2} / 2$. The antitumor treatment was performed when the tumor volume reached around $30 \mathrm{~mm}^{3}$ (Fig. S3 in the Electronic Supplementary Material (ESM)).

\subsection{Animal experiment}

The tumor-bearing nude mice were randomly divided into four groups: (1) PBS; (2) free ICG solution with laser irradiation $(0.1 \mathrm{mg} / \mathrm{mL}, 80 \mu \mathrm{L})$; (3) and (4) ICG-NBs- ${ }_{2}$ solution without or with laser irradiation (equivalent ICG concentration: $0.1 \mathrm{mg} / \mathrm{mL}$, $80 \mu \mathrm{L}$ ). A $80 \mu \mathrm{L}$ amount of PBS, free ICG, or ICG-NBs-O ${ }_{2}$ solution was intratumorally injected into the nude mice bearing Cal27 tumor. After intratumoral injection, the tumor sites of mice were irradiated by a NIR laser $\left(808 \mathrm{~nm}, 0.5 \mathrm{~W} / \mathrm{cm}^{2}\right)$ for $10 \mathrm{~min}$. Simultaneously, an infrared thermal imaging camera (TESTO 869, Germany) was used to continuously monitor the laser-induced temperature elevation of irradiation sites. Then the mouse body weight and tumor size were recorded continuously for 14 days. After the antitumor therapy, the mice were killed to harvest the major organs (heart, liver, spleen, lung, and kidney) and tumors. The excised organs and tumors were sliced and stained with hematoxylin and eosin (H\&E). Finally, the slices were observed and photographed using a digital microscope for histological analysis.

\subsection{Statistical analysis}

All data are expressed as mean \pm standard deviation (SD). Statistical evaluations were based on Student's $t$ tests or one-way analysis of variance (ANOVA).

\section{Results and discussion}

\subsection{Preparation and characterization of ICG-NBs- $\mathrm{O}_{2}$}

The free NBs and ICG-NBs- $\mathrm{O}_{2}$ solution were successively fabricated by using the repeated compression and self-assembly method [36,37]. The hydrodynamic size and zeta potential of free $\mathrm{NBs}$ and ICG-NBs- $\mathrm{O}_{2}$ in the aqueous solution were determined by the Zetasizer Nano ZS90 at room temperature. The average size and zeta potential of free NBs and ICG-NBs- $\mathrm{O}_{2}$ can be assessed by calculating the arithmetical mean values of three replications. As illustrated in Fig. 1(a), the size distributions of free NBs and ICGNBs- $\mathrm{O}_{2}$ both behaved according to gaussian distributions and peaked at 190 and $220 \mathrm{~nm}$, respectively. The average sizes of free $\mathrm{NBs}$ and ICG-NBs-O $\mathrm{O}_{2}$ were 198.9 and $221.6 \mathrm{~nm}$ with polydispersity indices of 0.159 and 0.109 , respectively, indicating that the average size of free NBs increased by $20 \mathrm{~nm}$ after assembled with ICG molecules. Furthermore, Fig. 1(b) shows that the average zeta potentials of free NBs and ICG-NBs- $\mathrm{O}_{2}$ were estimated to be -40.5 and $-39 \mathrm{mV}$, respectively. The high negative zeta potentials of both free NBs and ICG-NBs-O 2 reflected good colloidal stability. The results are consistent with other nanobubbles reported in Ref. [40]. TEM image further revealed that the ICG-NBs- $\mathrm{O}_{2}$ sized about $200 \mathrm{~nm}$ and appeared generally as spherical shape (Fig. 1(c)), which agreed well with the DLS measurements. To investigate the dissolved oxygen levels in ultrapure water, ultrapure water filled with oxygen (water- $\mathrm{O}_{2}$ ), free NBs- $\mathrm{O}_{2}$ and ICG-NBs- $\mathrm{O}_{2}$ aqueous solution, oxygen contents were assessed and illustrated in Fig. 1(d). The oxygen contents of free NBs- $\mathrm{O}_{2}$ and ICG-NBs- $\mathrm{O}_{2}$ aqueous solution are almost twice as that of water- $\mathrm{O}_{2}$ and 3.2-fold as that of ultrapure water, which may increase the oxygen supply during PDT treatments.

\subsection{Aqueous solution stability}

The stability of ICG-NBs- $\mathrm{O}_{2}$ in aqueous solution was evaluated at ICG concentration of $10 \mu \mathrm{g} / \mathrm{mL}$. Compared with the free NBs, the size and zeta potential stability assay of ICG-NBs- $\mathrm{O}_{2}$ solution were performed for 4 days when stored at $4{ }^{\circ} \mathrm{C}$. As indicated in Fig. 1(e), the average sizes of ICG-NBs- $\mathrm{O}_{2}$ and free NBs- $\mathrm{O}_{2}$ kept about 220 and $200 \mathrm{~nm}$ over 4 days, respectively. Furthermore, the average 

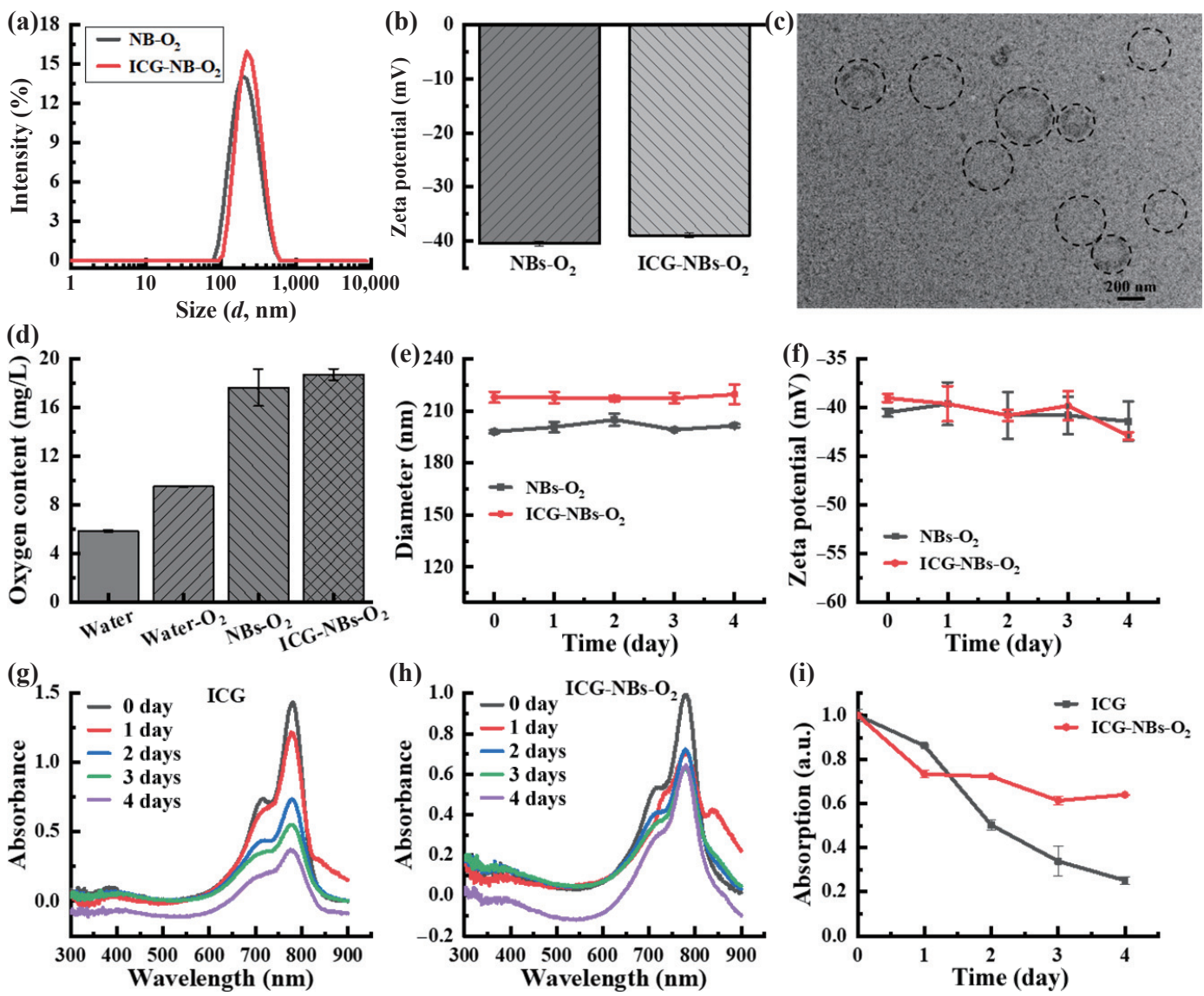

Figure 1 Characterizations of ICG-NBs- $\mathrm{O}_{2}$ : (a) intensity distribution of free $\mathrm{NBs}-\mathrm{O}_{2}$ and ICG-NBs- $\mathrm{O}_{2}$ in the aqueous solution; (b) average zeta potential of free NBs$\mathrm{O}_{2}$ and ICG-NBs- $\mathrm{O}_{2}$ in the aqueous solution; (c) TEM image of ICG-NBs- $\mathrm{O}_{2}$; (d) oxygen contents in water, water- $\mathrm{O}_{2}$, free NBs- $\mathrm{O}_{2}$, and ICG-NBs- $\mathrm{O}_{2}$ aqueous

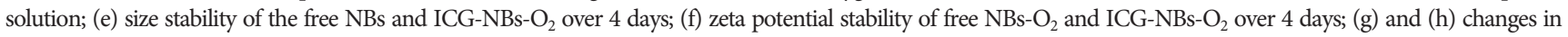
UV-Vis-NIR absorption spectra of ICG and ICG-NBs- $\mathrm{O}_{2}$ over 4 days; (i) normalized UV-Vis-NIR absorption spectra for ICG and ICG-NBs-O ${ }_{2}$.

zeta potential of ICG-NBs- $\mathrm{O}_{2}$ and free $\mathrm{NBs}-\mathrm{O}_{2}$ remained similar values $(-40 \mathrm{mV})$ for 4 days, suggesting good colloidal stability (Fig. 1(f)). Meanwhile, the stability of ICG-NBs- ${ }_{2}$ solution was also demonstrated by UV-Vis-NIR absorption spectra. As illustrated in Figs. 1(g) and 1(h), the typical absorption peaks of free ICG and ICG-NBs- $\mathrm{O}_{2}$ solution are almost the same. As can be seen from Fig. 1(i), after 1 day, the absorption peak intensity of ICG-NBs- $\mathrm{O}_{2}$ and free ICG solution remained at 0.73 and 0.86 , respectively. After 2 days, the absorption peak intensity of ICGNBs- $\mathrm{O}_{2}$ solution stayed at 0.72 while free ICG degraded to 0.50 . After 4 days, the absorption peak intensity of free ICG solution sharply decreased to 0.25 of the initial value. In contrast, the ICG$\mathrm{NBs}-\mathrm{O}_{2}$ solution remained at 0.64 . The above results indicated

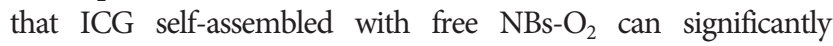
enhance the stability of ICG aqueous solution, which were similar to the results in our previous work [36].

\section{3 ${ }^{1} \mathrm{O}_{2}$ detection and photothermal effect}

\subsection{1 ${ }^{1} \mathrm{O}_{2}$ generation of ICG-NBs filled with different gases}

In order to gain insight into the effects of different gases on ${ }^{1} \mathrm{O}_{2}$ generation, the ${ }^{1} \mathrm{O}_{2}$ QY values of ICG-NBs (ICG concentration: 10 $\mu \mathrm{g} / \mathrm{mL}$ ) filled with different gases were examined. The DPBF was used as a ${ }^{1} \mathrm{O}_{2}$ sensor to assess the capability of ICG-NBs as potential PDT agents under laser irritation. The absorption of DPBF was recorded with time under laser irradiation (wavelength: $808 \mathrm{~nm}$, power density: $1 \mathrm{~W} / \mathrm{cm}^{2}$ ). Figures 2(a)-2(e) show the absorption spectra of DPBF in presence of ICG and ICG-NBs filled with $\mathrm{SF}_{6}, \mathrm{~N}_{2}$, Xe and $\mathrm{O}_{2}$, respectively. It can be seen that the ICG-NBs, especially filled with $\mathrm{O}_{2}$, displayed much faster generation rate of ${ }^{1} \mathrm{O}_{2}$ than free ICG solution when irradiated with laser after 0, 2, 4, 6, 8, 10, and $15 \mathrm{~min}$. As illustrated in Fig. 2(f), the absorbance of DPBF at $420 \mathrm{~nm}$ in water showed negligible decrease with time. After irradiated $15 \mathrm{~min}$, the absorbance intensity at $420 \mathrm{~nm}$ of DPBF in free ICG solution remained at 0.89 of the initial. In contrast, the absorbance intensity of ICG-NBs solution filled with $\mathrm{SF}_{6}, \mathrm{~N}_{2}, \mathrm{Xe}$, and $\mathrm{O}_{2}$ decreased to $0.72,0.70,0.78$, and 0.49 , respectively. The results indicated that ICG-NBs can significantly enhance the capability of ${ }^{1} \mathrm{O}_{2}$ generation. Among these ICG-NBs, ICG-NBs- $\mathrm{O}_{2}$ showed the best capability of ${ }^{1} \mathrm{O}_{2}$ generation.

\subsection{2 ${ }^{1} \mathrm{O}_{2}$ generation of ICG assembled with different concentrations of free $\mathrm{NBs}-\mathrm{O}_{2}$}

To optimize the ${ }^{1} \mathrm{O}_{2}$ production of ICG-NBs- $\mathrm{O}_{2}$, ICG assembling

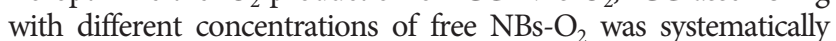
investigated. The NBs-R1 and NBs-R2 solution were prepared by

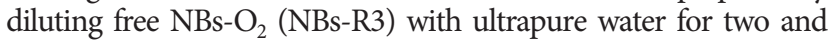
four times, respectively. Then, ICG-NBs-R1, ICG-NBs-R2, and ICG-NBs-R3 were prepared by assembling ICG solution (concentration: $10 \mu \mathrm{g} / \mathrm{mL}$ ) with NBs-R1, NBs-R2, and NBs-R3, respectively. As can be seen from Figs. 3(a)-3(c), the absorption intensity decreased with the increasing concentration of free NBs$\mathrm{O}_{2}$. The absorption spectra of ICG-NBs-O 2 (R1 to R3) showed the same trend (Figs. 3(d) and 3(e), Fig. 2(e)). The absorbance values of DPBF were normalized at $420 \mathrm{~nm}$ as shown in Fig. 3(f). The decomposition rate constants of DPBF by ICG-NBs- $\mathrm{O}_{2}$ and ICG were determined by the absorption losses of DPBF with time (Fig. 3(f)). According to the above-mentioned Eq. (1), the ${ }^{1} \mathrm{O}_{2}$ QY of ICG-NBs-O 2 (ICG-NBs-R3) can be estimated to be $1.6 \%$ (Fig. S1 in the ESM, using ICG as the standard reference with ${ }^{1} \mathrm{O}_{2} \mathrm{QY}$ of $0.2 \%$ [39]), which was 8 times as high as that of free ICG solution.

Moreover, the comparison of photothermal effect between the 

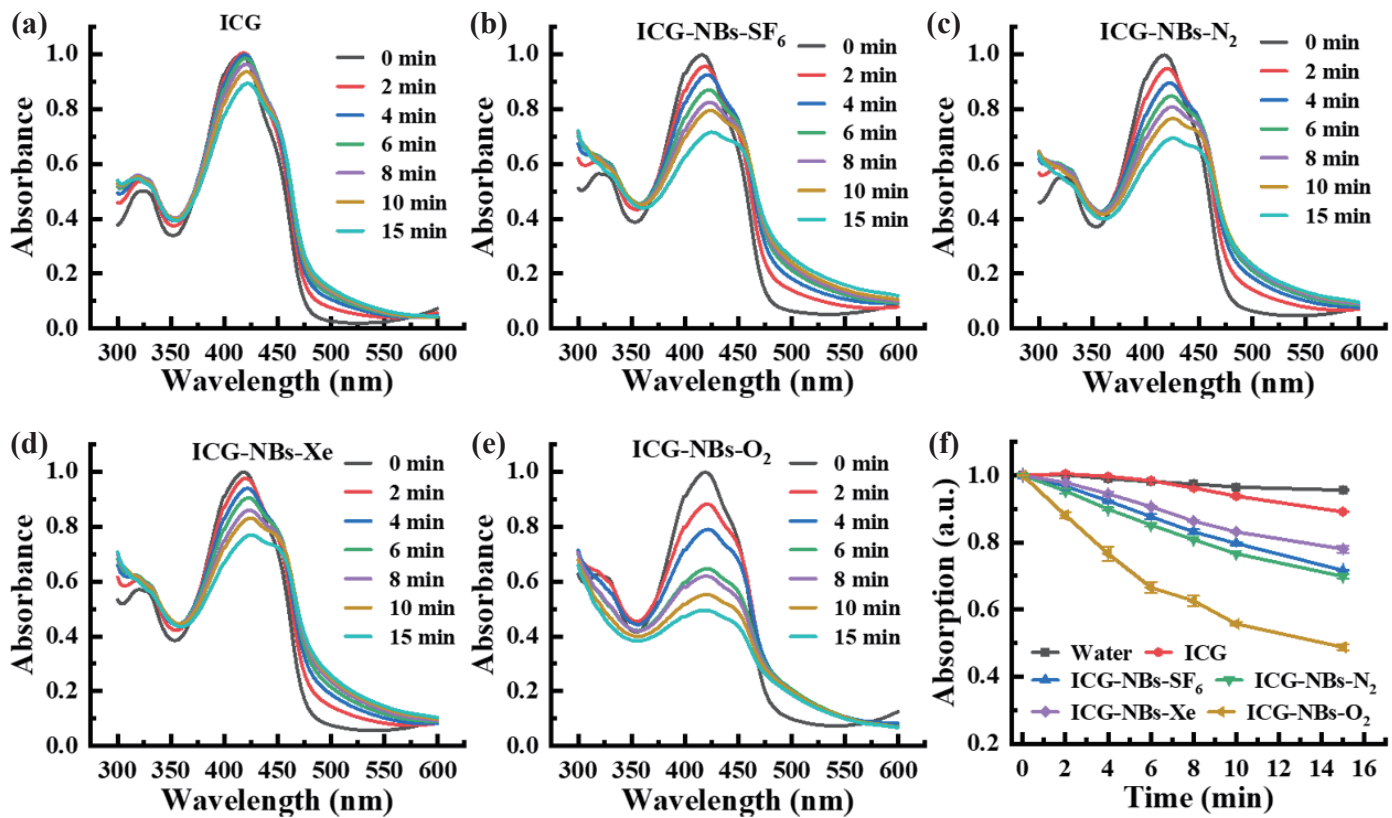

Figure 2 The absorption decrease of DPBF in presence of (a) free ICG; (b)-(e) ICG-NBs filled with $\mathrm{SF}_{6}, \mathrm{~N}_{2}, \mathrm{Xe}_{\mathrm{e}}$ and $\mathrm{O}_{2}$, respectively, after $0,2,4,6,8,10$, and 15 min laser irradiation. (f) Normalized absorbance values at $420 \mathrm{~nm}$ of free ICG and ICG-NBs.
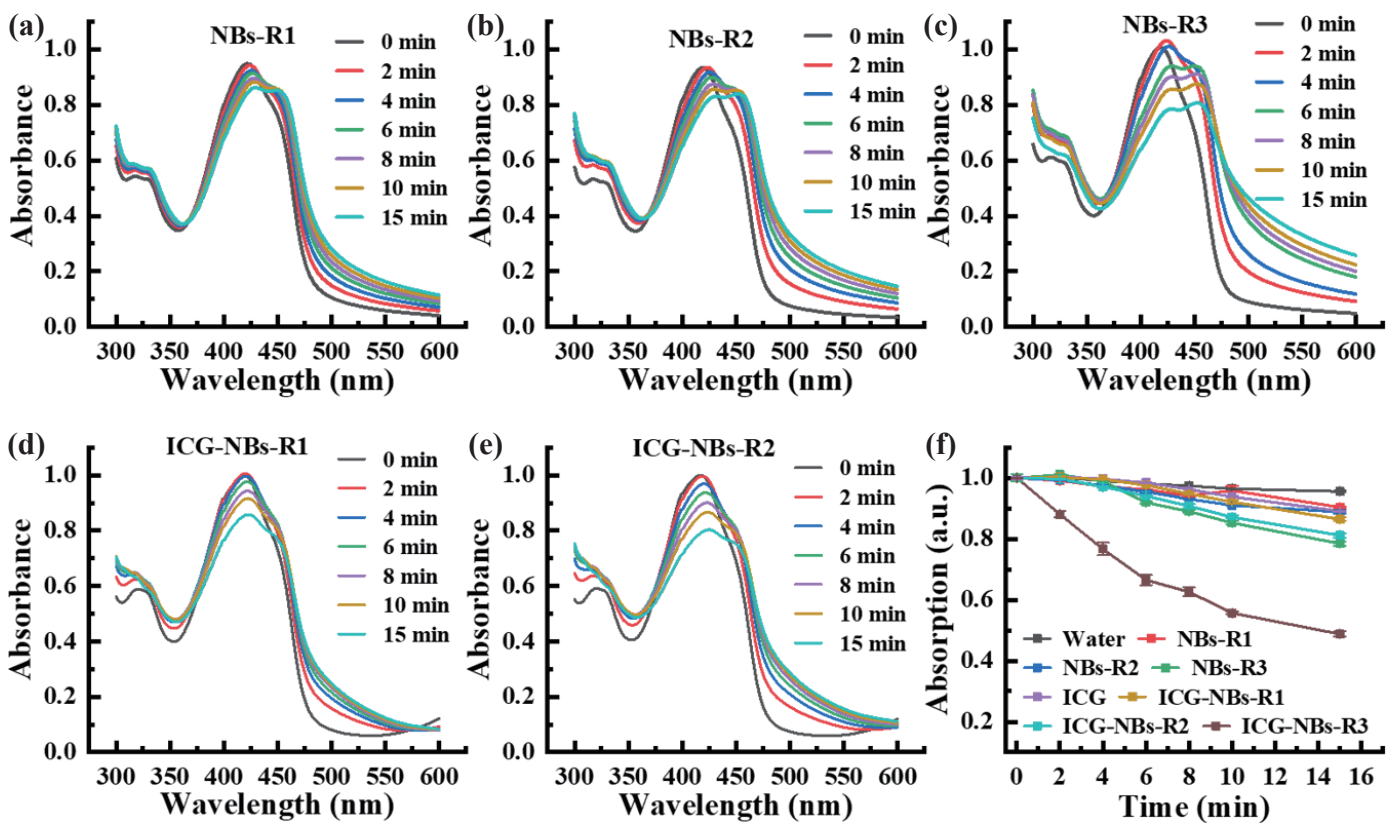

Figure 3 The absorption decrease of DPBF in presence of (a)-(c) different concentration of free NBs- $\mathrm{O}_{2}$; (d) and (e) ICG assembled with different concentration of ICG-NBs- $\mathrm{O}_{2}$. (f) Normalized absorbance values at $420 \mathrm{~nm}$ of free ICG, free $\mathrm{NBs}-\mathrm{O}_{2}$, and ICG-NBs- $\mathrm{O}_{2}$.

free ICG and ICG-NBs- $\mathrm{O}_{2}$ under laser irradiation was also accomplished and the results were shown in the ESM. The ICGNBs- $\mathrm{O}_{2}$ and free ICG solution displayed similar temperature rise with time (Fig. S2 in the ESM). The result suggested that ICG molecules assembled onto free $\mathrm{NBs}-\mathrm{O}_{2}$ did not change the photothermal effect of ICG. The high ${ }^{1} \mathrm{O}_{2}$ QY and significant stability of ICG-NBs- $\mathrm{O}_{2}$ aqueous solution indicated the potential application as a photosensitizer (PS) agent in PDT treatment.

\subsection{In vitro cellular uptake}

Next, the intracellular uptake ability of ICG-NBs- $\mathrm{O}_{2}$ was estimated. The human tongue cancer cells $\mathrm{Cal} 27$ were incubated with free ICG or ICG-NBs- $\mathrm{O}_{2}$ solution at the same concentration of ICG $(10 \mu \mathrm{g} / \mathrm{mL})$ for $24 \mathrm{~h}$. Compared with free ICG solution, the cellular uptake and distribution of ICG-NBs- $\mathrm{O}_{2}$ solution were visualized by CLSM. From Fig. 4, cancer cells Cal27 treated by ICG-NBs- $\mathrm{O}_{2}$ solution displayed significantly higher fluorescence intensity than that of cells treated by free ICG solution. Furthermore, the red fluorescence can be observed both in cytoplasm and nucleus, which demonstrated the extensive distribution of ICG in the cells. The results indicated that the free NBs- $\mathrm{O}_{2}$ had strong ability to carry ICG and to mediate ICG endocytosis.

\subsection{Intracellular ${ }^{1} \mathrm{O}_{2}$ generation}

DCFH-DA probe was used as the ${ }^{1} \mathrm{O}_{2}$ sensor to evaluate the capability of ICG-NBs- $\mathrm{O}_{2}$ as potential PDT agents in tumor cell under laser irritation. DCFH-DA can be oxidized by ${ }^{1} \mathrm{O}_{2}$ into a green fluorescent compound (2,7-dichlorofluoresein, DCF). So, Cal27 cells were treated with DCFH-DA, and then the green fluorescence of DCF was examined with CLSM (Fig. 5). Without laser irradiation, as shown in Fig. 5(a), the weak fluorescence can be detected in cytoplasm, which indicated the negligible level of ${ }^{1} \mathrm{O}_{2}$ generation. However, with laser irradiation, the obvious green 

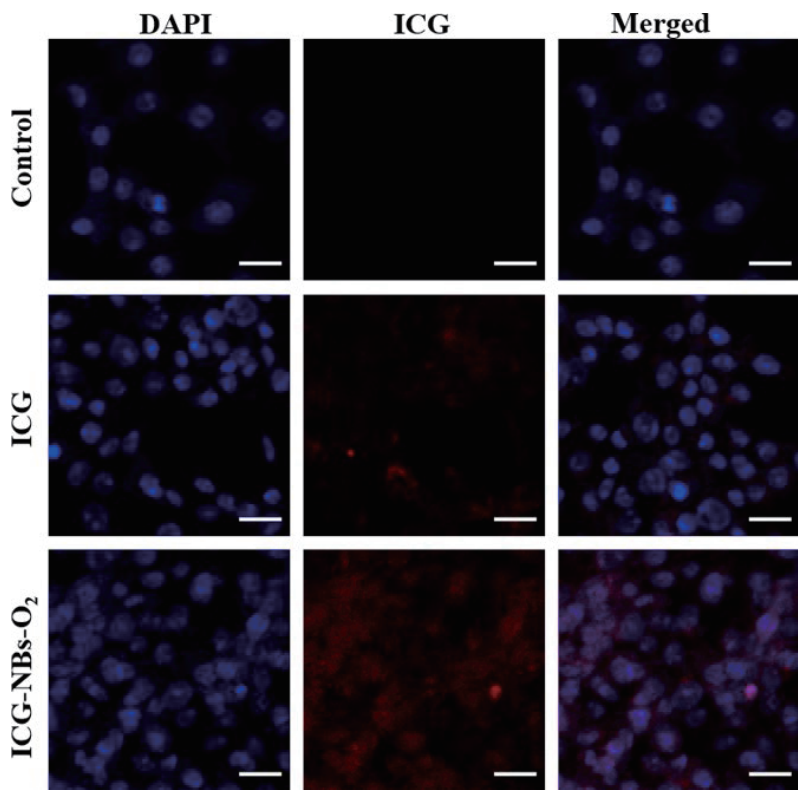

Figure 4 Confocal images for endocytosis of free ICG and ICG-NBs- $\mathrm{O}_{2}$. Blue and red represent the fluorescence of DAPI and ICG, respectively. Scale bar = $25 \mu \mathrm{m}$.
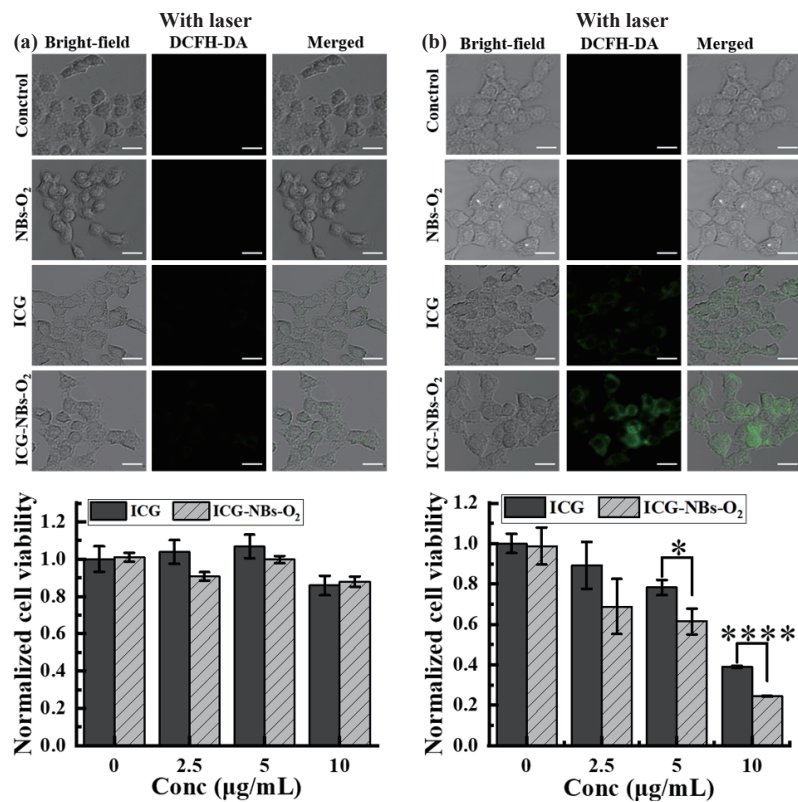

Figure 5 The intracellular ROS generation analysis and cytotoxicity of ICGNBs- $\mathrm{O}_{2}$. Confocal images of Cal27 cells after treatment with free NBs, ICG, and ICG-NBs- $\mathrm{O}_{2}$, without (a) and with (b) NIR laser irradiation, scale bar $=25 \mu \mathrm{m}$; (c) Cal27 cell viability incubated with ICG and ICG-NBs-O $\mathrm{O}_{2}$ after $24 \mathrm{~h}$ under various ICG concentrations; (d) viabilities of $\mathrm{Cal} 27$ cells after treatment with different ICG concentrations of free ICG and ICG-NBs- $\mathrm{O}_{2}$ subjected to laser irradiation.

fluorescence appeared in Cal27 cells treated with free ICG and ICG-NBs- $\mathrm{O}_{2}$ solution (Fig. 5(b)). The strong fluorescence intensity of ICG-NBs- $\mathrm{O}_{2}$ solution treated cells indicated the remarkably higher level of ${ }^{1} \mathrm{O}_{2}$ than free ICG solution. The results suggested the feasibility of ICG-NBs- $\mathrm{O}_{2}$ as a PS agent in PDT treatments.

The cytotoxicity of ICG-NBs- $\mathrm{O}_{2}$ and free ICG to human tongue cancer cells Cal27 was estimated by CCK- 8 assay. As shown in Fig. 5(c), the cell viability showed a slight decline with the increase of ICG concentration. The viability of Cal27 treated with ICG-NBs- $\mathrm{O}_{2}$ solution with ICG concentration of $10 \mu \mathrm{g} / \mathrm{mL}$ was maintained about $88 \%$, which demonstrated the excellent biocompatibility of the ICG-NBs- $\mathrm{O}_{2}$. Compared with free ICG solution, the ablation effect of ICG-NBs- $\mathrm{O}_{2}$ exposed to an NIR laser $\left(808 \mathrm{~nm}, 1 \mathrm{~W} / \mathrm{cm}^{2}\right)$ was evaluated with CCK-8 assay. To test the PDT effect alone, the cells were irradiated at $4{ }^{\circ} \mathrm{C}$ with the NIR laser for $5 \mathrm{~min}$. As shown in Fig. 5(d), under NIR laser irradiation, the cell viability of $\mathrm{Cal} 27$ exhibited a dose-dependent decrease both in the free ICG and ICG-NBs- $\mathrm{O}_{2}$ solution. Especially, the ICG-NBs- $\mathrm{O}_{2}$ solution exposed to laser showed much higher cytotoxicity than the free ICG solution. The cell killing ability increased by $20 \%$ (Fig. 5(d)). The significant cell killing ability was ascribed to the enhanced photostability and oxygen environment of ICG-NBs- $\mathrm{O}_{2}$ solution.

\subsection{Antitumor effect of ICG-NBs- $\mathrm{O}_{2}$ in vivo}

In vivo, antitumor effect of ICG-NBs- $\mathrm{O}_{2}$ was investigated by establishing the tumor model in nude mice. An infrared thermal imaging camera was employed to record the body temperature of tumor-bearing mice during NIR laser irradiation. As expected, the mouse body temperature at the tumor site in all groups increased with the increment of radiation time (Figs. 6(a) and 6(b)). In the control group, the temperature at tumor site increased about $3{ }^{\circ} \mathrm{C}$ under laser irradiation for $10 \mathrm{~min}$. In contrast, in the case of free ICG and ICG-NBs- $\mathrm{O}_{2}$-treated groups, the maximum temperature at tumor site reached around $42{ }^{\circ} \mathrm{C}$ with a temperature increase about $14{ }^{\circ} \mathrm{C}$ under $10 \mathrm{~min}$ of laser irradiation. Free ICG and ICG$\mathrm{NBs}-\mathrm{O}_{2}$ exhibited equivalent hyperthermia, indicating similar photothermal conversion ability for tumor ablation. To demonstrate the PDT efficacy of ICG-NBs- $\mathrm{O}_{2}$ on tumor suppression, the tumor volume (Fig. 6(c)) and mouse body weight were recorded every two days. In the control group and ICG-NBs$\mathrm{O}_{2}$ treated group (without laser irradiation), compared with the initial tumor size on day 1 , the relative tumor volume on day 14 rapidly increased to 3.9 and 4.3 -fold, respectively, indicating insignificant tumor inhibition effect of ICG-NBs- $\mathrm{O}_{2}$ without laser irradiation. In free ICG treated group (with laser irradiation) group, the relative tumor volume increased to 2.4 -fold on day 14 , reflecting definite effect of free ICG on antitumor. In contrast, in the ICG-NBs- $\mathrm{O}_{2}$ treated group (with laser irradiation), the relative tumor volume gradually decreased to 0.56 of the initial tumor size, suggesting a notable tumor inhibition effect of ICG-NBs- $\mathrm{O}_{2}$. The photographs of the excised tumors shown in Fig. 6(e) further verified these results. On consideration of the equivalent photothermal effects of free ICG with laser and ICG-NBs- $\mathrm{O}_{2}$ on tumor, the differences in relative tumor volume on day 14 were attributed to their PDT efficacy. Benefited from the enhanced solution stability of ICG molecules and improved oxygen contents in tumor environment, the PDT therapeutic effect of ICG-NBs- $\mathrm{O}_{2}$ on tumor was significantly enhanced. Additionally, the body weight of the mice did not show obvious difference in all the groups on day 14, which preliminarily verified the

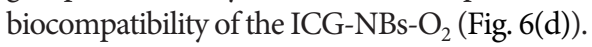

$\mathrm{H} \& \mathrm{E}$ staining was used to examine the deterioration of tumor cells after the various treatments. As illustrated in Fig. 6(f), in the control group, most tumor cells remained the regular morphology and no histopathological abnormalities was observed. However, in ICG-NBs- $\mathrm{O}_{2}$ treated group (with laser radiation), the tumor cells exhibited irregular shape and obvious karyorrhexis, pyknosis, and karyolysis can be found, indicating the severe tumor cell ablation. Moreover, the TUNEL staining (Fig. 6(g)) also verified that severe tumor cell apoptosis occurred in ICG-NBs- $\mathrm{O}_{2}$ treated group (with laser radiation). The above results demonstrated the enhanced therapeutic effect of ICG-NBs- $\mathrm{O}_{2}$ compared with free ICG.

\subsection{Biocompatibility evaluation}

To investigate the histocompatibility of ICG-NBs- $\mathrm{O}_{2}$, the major 
(a)

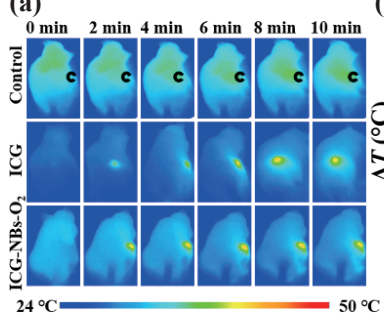

(b)

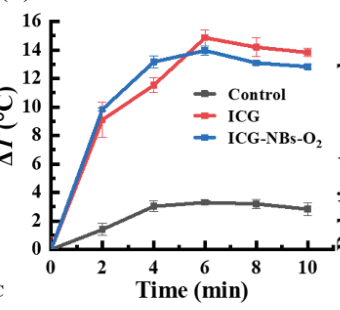

(c)

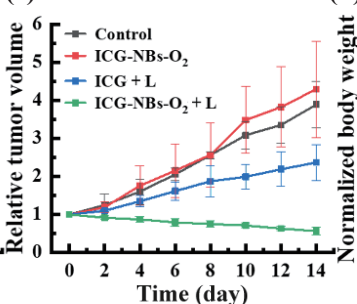

(d)

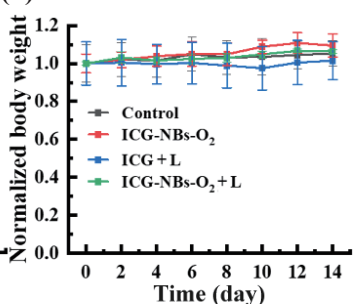

(e)

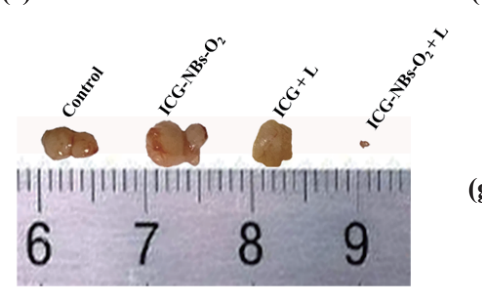

(f)
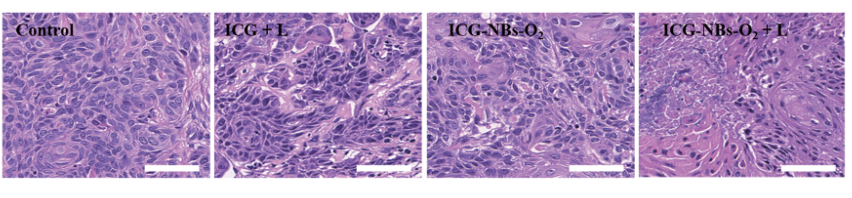

(g)
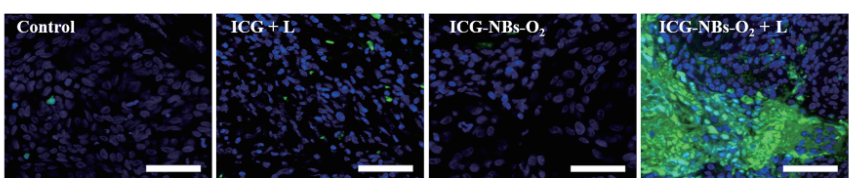

Figure 6 (a) Photothermal imagings of tumor-bearing mice under NIR laser irradiation $\left(808 \mathrm{~nm}, 0.5 \mathrm{~W} / \mathrm{cm}^{2}\right)$ for $10 \mathrm{~min}$; (b) variations of the peak temperature at tumor site corresponding to (a); (c) tumor volume evolution over 14 days after different treatments; (d) normalized mouse body weight over 14 days in the different groups after various treatments; (e) photographs of the excised tumors after various treatments; (f) H\&E staining and (g) TUNEL staining (scale bar: 50 $\mu$ ), blue and green represent the fluorescence of DAPI and TUNEL staining, respectively.

organs (heart, liver, spleen, lung, and kidney) of every group of treated mice were excised and collected on day 14 for H\&E staining (Fig. 7). There was no observable inflammation or lesion abnormalities noticed in any organs of all mouse groups. Furthermore, after administration of ICG-NBs- $\mathrm{O}_{2}$, the blood biochemical analysis was performed to obtain the complete count of key blood components. As shown in Fig. S4 in the ESM, compared with the control group, there were no significant differences in the hematological indexes in the treated mice. The results indicated the good biosafety and reliable therapeutic effect of ICG-NBs- $\mathrm{O}_{2}$.

\section{Conclusions}

In summary, a facile route to fabricate ICG-NBs- $\mathrm{O}_{2}$ solution with average size of $220 \mathrm{~nm}$ was developed by assembling ICG molecules with free $\mathrm{NBs}-\mathrm{O}_{2}$. The ICG-NBs- $\mathrm{O}_{2}$ aqueous solution exhibited outstanding stability of aqueous solution. After 4 days, the absorption peak intensity of free ICG solution decreased to 0.25 while the ICG-NBs- $\mathrm{O}_{2}$ solution remained at 0.64 of the initial value. Furthermore, the ICG-NBs- $\mathrm{O}_{2}$ can greatly improve oxygen contents in aqueous solution. The oxygen content of ICG-NBs- $\mathrm{O}_{2}$ aqueous solution was 3.2-fold as that of ultrapure water. Therefore, benefited from the significantly enhanced stability and oxygen contents of aqueous solution, the ${ }^{1} \mathrm{O}_{2}$ QY of ICG-NBs- $\mathrm{O}_{2}$ solution was 8 times as that of the free ICG solution, suggesting the exellent photodynamic properties. Subsequently, the therapeutic effect of ICG-NBs- $\mathrm{O}_{2}$ solution was examined systematically based on tumor cell and tumor model. Compared with the initial tumor size on day 1 , the relative tumor volume of mice treated with ICG increased to 2.4 times while the relative tumor volume treated with ICG-NBs- $\mathrm{O}_{2}$ decreased to 0.56 on day 14 , reflecting the outstanding PDT efficacy of ICG-NBs- $\mathrm{O}_{2}$. Moreover, the ICG$\mathrm{NBs}-\mathrm{O}_{2}$ displayed great biosafety on the mouse model, indicating a promising potential for tumor treatment in clinical application.
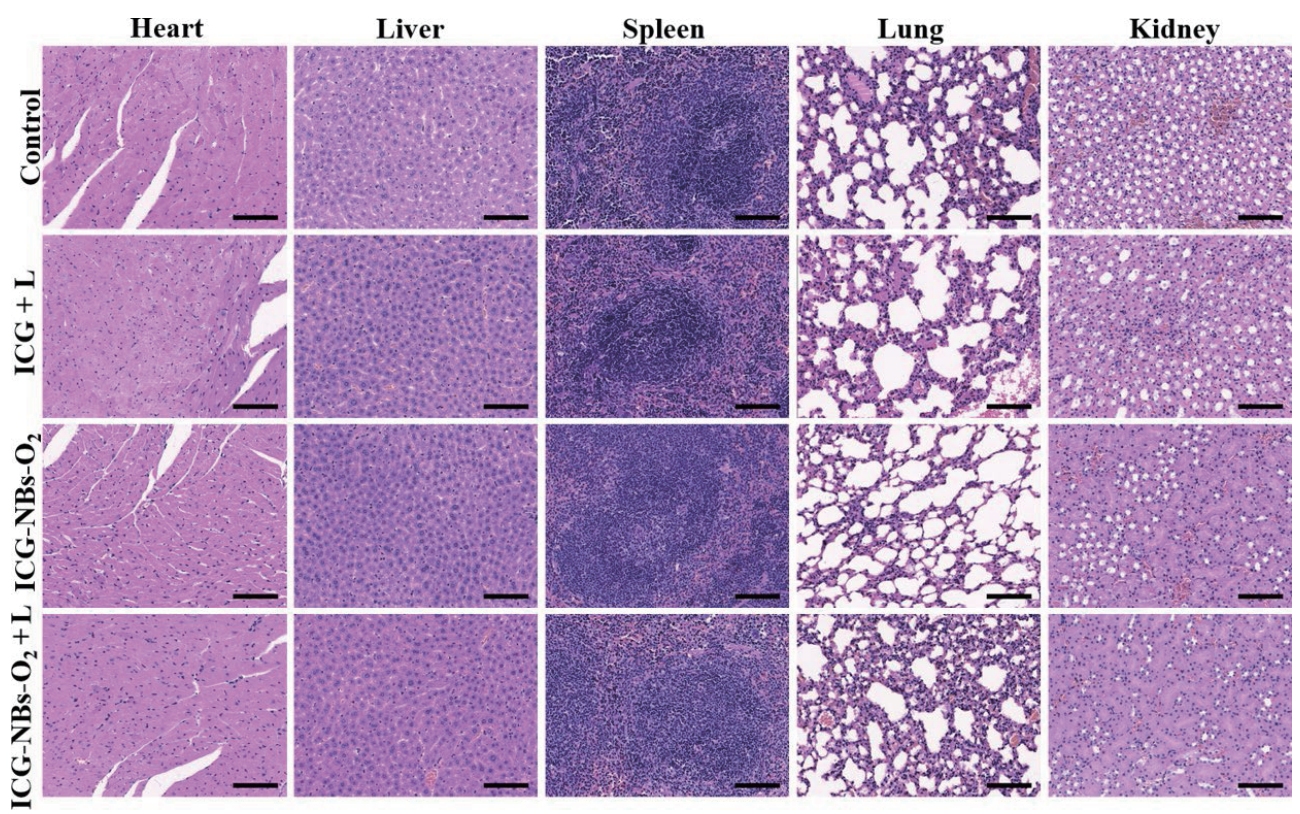

Figure 7 H\&E staining of the major organs isolated from the mice after various treatments (scale bar: $100 \mu \mathrm{m}$ ). 
We believe that this work will open new perspectives for the clinical application of ICG in PDT.

\section{Acknowledgements}

This investigation was financially funded by the projects of the National Key Research and Development Program of China (No. 2017YFA0104302), the National Natural Science Foundation of China (Nos. 51832001, 61821002, and 81971750), and the Jiangsu Planned Projects for Postdoctoral Research Funds (No. 2021K601C).

Electronic Supplementary Material: Supplementary material (measurements of the singlet oxygen quantum yield of ICG-NBs$\mathrm{O}_{2}$, time-dependent temperature changes during the laser irradiation, photographs of Cal27 tumor-bearing nude mice and complete blood count of health male balb/c mice analysis ) is available in the online version of this article at https://doi.org/10.1007/s12274-022-4085-0.

\section{References}

[1] Shibu, E. S.; Hamada, M.; Murase, N.; Biju, V. Nanomaterials formulations for photothermal and photodynamic therapy of cancer. J. Photochem. Photobiol. C Photochem. Rev. 2013, 15, 53-72.

[2] Liu, J. J.; Liu, K.; Feng, L. Z.; Liu, Z.; Xu, L. G. Comparison of nanomedicine-based chemotherapy, photodynamic therapy and photothermal therapy using reduced graphene oxide for the model system. Biomater. Sci. 2017, 5, 331-340.

[3] Hak, A.; Shinde, V. R.; Rengan, A. K. A review of advanced nanoformulations in phototherapy for cancer therapeutics. Photodiagnosis Photodyn. Ther. 2021, 33, 102205.

[4] Yan, J. X.; Wang, C. L.; Jiang, X. M.; Wei, Y. Q.; Wang, Q.; Cui, K. L.; Xu, X.; Wang, F.; Zhang, L. Application of phototherapeuticbased nanoparticles in colorectal cancer. Int. J. Biol. Sci. 2021, 17, 1361-1381.

[5] Fan, H. Y.; Zhu, Z. L.; Zhang, W. L.; Yin, Y. J.; Tang, Y. L.; Liang, X. H.; Zhang, L. Light stimulus responsive nanomedicine in the treatment of oral squamous cell carcinoma. Eur. J. Med. Chem. 2020, 199, 112394.

[6] Zou, L. L.; Wang, H.; He, B.; Zeng, L. J.; Tan, T.; Cao, H. Q.; He, X. Y.; Zhang, Z. W.; Guo, S. R.; Li, Y. P. Current approaches of photothermal therapy in treating cancer metastasis with nanotherapeutics. Theranostics 2016, 6, 762-772.

[7] Martins, W. K.; Belotto, R.; Silva, M. N.; Grasso, D.; Suriani, M. D.; Lavor, T. S.; Itri, R.; Baptista, M. S.; Tsubone, T. M. Autophagy regulation and photodynamic therapy: Insights to improve outcomes of cancer treatment. Front. Oncol. 2021, 10, 610472.

[8] Ethirajan, M.; Chen, Y. H.; Joshi, P.; Pandey, R. K. The role of porphyrin chemistry in tumor imaging and photodynamic therapy. Chem. Soc. Rev. 2011, 40, 340-362.

[9] Dias, L. D.; Bagnato, V. S. An update on clinical photodynamic therapy for fighting respiratory tract infections: A promising tool against COVID-19 and its co-infections. Laser Phys. Lett. 2020, 17, 083001.

[10] Stájer, A.; Kajári, S.; Gajdács, M.; Musah-Eroje, A.; Baráth, Z. Utility of photodynamic therapy in dentistry: Current concepts. Dent. J. 2020, 8, 43.

[11] Kwon, N.; Kim, H.; Li, X. S.; Yoon, J. Supramolecular agents for combination of photodynamic therapy and other treatments. Chem. Sci. 2021, 12, 7248-7268.

[12] Hong, E. J.; Choi, D. G.; Shim, M. S. Targeted and effective photodynamic therapy for cancer using functionalized nanomaterials. Acta Pharm. Sin. B 2016, 6, 297-307.

[13] Chilakamarthi, U.; Giribabu, L. Photodynamic therapy: Past, present and future. Chem. Rec. 2017, 17, 775-802.

[14] Dolmans, D. E. J. G. J.; Fukumura, D.; Jain, R. K. Photodynamic therapy for cancer. Nat. Rev. Cancer 2003, 3, 380-387.

[15] Kawczyk-Krupka, A.; Bugaj, A. M.; Latos, W.; Zaremba, K.;
Wawrzyniec, K.; Kucharzewski, M.; Sieroń, A. Photodynamic therapy in colorectal cancer treatment-The state of the art in preclinical research. Photodiagnosis Photodyn. Ther. 2016, 13, 158-174.

[16] Mu, C. N.; Wang, W.; Wang, J.; Gong, C.; Zhang, D. M.; Zhang, X. $\mathrm{X}$. Probe-free direct identification of type I and type II photosensitized oxidation using field-induced droplet ionization mass spectrometry. Angew. Chem., Int. Ed. 2020, 59, 21515-21519.

[17] Estébanez, S.; Lorente, C.; Kaufman, T. S.; Larghi, E. L.; Thomas, A. H.; Serrano, M. P. Photophysical and photochemical properties of 3-methylpterin as a new and more stable pterin-type photosensitizer. Photochem. Photobiol. 2018, 94, 881-889.

[18] Baptista, M. S.; Cadet, J.; Di Mascio, P.; Ghogare, A. A.; Greer, A.; Hamblin, M. R.; Lorente, C.; Nunez, S. C.; Ribeiro, M. S.; Thomas, A. H. et al. Type I and type II photosensitized oxidation reactions: Guidelines and mechanistic pathways. Photochem. Photobiol. 2017, 93, 912-919.

[19] Figueiró Longo, J. P.; Lozzi, S. P.; Simioni, A. R.; Morais, P. C.; Tedesco, A. C.; Azevedo, R. B. Photodynamic therapy with aluminum-chloro-phtalocyanine induces necrosis and vascular damage in mice tongue tumors. J. Photochem. Photobiol. B Biol. 2009, 94, 143-146.

[20] Jayme, C. C.; Calori, I. R.; Freire Cunha, E. M.; Tedesco, A. C. Evaluation of aluminum phthalocyanine chloride and DNA interactions for the design of an advanced drug delivery system in photodynamic therapy. Spectrochim. Acta A Mol. Biomol. Spectrosc. 2018, 201, 242-248.

[21] Bicalho, L. S.; Longo, J. P. F.; Cavalcanti, C. E.; Simioni, A. R.; Bocca, A. L.; de Almeida Santos, M. D. F. M.; Tedesco, A. C.; Azevedo, R. B. Photodynamic therapy leads to complete remission of tongue tumors and inhibits metastases to regional lymph nodes. $J$. Biomed. Nanotechnol. 2013, 9, 811-818.

[22] Yang, S. J.; Lin, C. F.; Kuo, M. L.; Tan, C. T. Photodynamic detection of oral cancers with high-performance chitosan-based nanoparticles. Biomacromolecules 2013, 14, 3183-3191.

[23] Wang, X. H.; Yu, Y. X.; Cheng, K.; Yang, W.; Liu, Y. A.; Peng, H. S. Polylysine modified conjugated polymer nanoparticles loaded with the singlet oxygen probe 1,3-diphenylisobenzofuran and the photosensitizer indocyanine green for use in fluorometric sensing and in photodynamic therapy. Microchim. Acta 2019, 186, 842.

[24] Shi, L. L.; Hu, F.; Duan, Y. K.; Wu, W. B.; Dong, J. Q.; Meng, X. J.; Zhu, X. Y.; Liu, B. Hybrid nanospheres to overcome hypoxia and intrinsic oxidative resistance for enhanced photodynamic therapy. ACS Nano 2020, 14, 2183-2190.

[25] Wan, Y. P.; Lu, G. H.; Zhang, J. F.; Wang, Z. Y.; Li, X. Z.; Chen, R.; Cui, X.; Huang, Z. M.; Xiao, Y. F.; Chelora, J. et al. A biocompatible free radical nanogenerator with real-time monitoring capability for high performance sequential hypoxic tumor therapy. Adv. Funct. Mater. 2019, 29, 1903436.

[26] Hu, J. J.; Lei, Q.; Zhang, X. Z. Recent advances in photonanomedicines for enhanced cancer photodynamic therapy. Prog. Mater. Sci. 2020, 114, 100685.

[27] Khan, S.; Sharifi, M.; Hasan, A.; Attar, F.; Edis, Z.; Bai, Q.; Derakhshankhah, H.; Falahati, M. Magnetic nanocatalysts as multifunctional platforms in cancer therapy through the synthesis of anticancer drugs and facilitated Fenton reaction. J. Adv. Res. 2021, 30, 171-184.

[28] Treger, J. S.; Priest, M. F.; Iezzi, R.; Bezanilla, F. Real-time imaging of electrical signals with an infrared FDA-approved dye. Biophys. $J$. 2014, 107, L09-L12.

[29] Sheng, Z. H.; Hu, D. H.; Xue, M. M.; He, M.; Gong, P.; Cai, L. T. Indocyanine green nanoparticles for theranostic applications. NanoMicro Lett. 2013, 5, 145-150.

[30] Zhang, R. R.; Schroeder, A. B.; Grudzinski, J. J.; Rosenthal, E. L.; Warram, J. M.; Pinchuk, A. N.; Eliceiri, K. W.; Kuo, J. S.; Weichert, J. P. Beyond the margins: Real-time detection of cancer using targeted fluorophores. Nat. Rev. Clin. Oncol. 2017, 14, 347-364.

[31] Vahrmeijer, A. L.; Hutteman, M.; van der Vorst, J. R.; van de Velde, C. J. H.; Frangioni, J. V. Image-guided cancer surgery using nearinfrared fluorescence. Nat. Rev. Clin. Oncol. 2013, 10, 507-518. 
[32] Guan, S. Y.; Weng, Y. Z. W.; Li, M. N.; Liang, R. Z.; Sun, C. H.; Qu, X. Z.; Zhou, S. Y. An NIR-sensitive layered supramolecular nanovehicle for combined dual-modal imaging and synergistic therapy. Nanoscale 2017, 9, 10367-10374.

[33] Wu, L.; Fang, S. T.; Shi, S.; Deng, J. Z.; Liu, B.; Cai, L. T. Hybrid polypeptide micelles loading indocyanine green for tumor imaging and photothermal effect study. Biomacromolecules 2013, 14, 3027-3033.

[34] Lee, C. H.; Cheng, S. H.; Wang, Y. J.; Chen, Y. C.; Chen, N. T.; Souris, J.; Chen, C. T.; Mou, C. Y.; Yang, C. S.; Lo, L. W. Nearinfrared mesoporous silica nanoparticles for optical imaging: Characterization and in vivo biodistribution. Adv. Funct. Mater. 2009, 19, 215-222.

[35] Patel, R. H.; Wadajkar, A. S.; Patel, N. L.; Kavuri, V. C.; Nguyen, K. T.; Liu, H. L. Multifunctionality of indocyanine green-loaded biodegradable nanoparticles for enhanced optical imaging and hyperthermia intervention of cancer. J. Biomed. Opt. 2012, 17, 046003.

[36] Yang, L.; Huang, B.; Chen, F.; Jin, J.; Qin, Z. G.; Yang, F.; Li, Y.;
$\mathrm{Gu}, \mathrm{N}$. Indocyanine green assembled nanobubbles with enhanced fluorescence and photostability. Langmuir 2020, 36, 12983-12989.

[37] Jin, J.; Feng, Z. Q.; Yang, F.; Gu, N. Bulk nanobubbles fabricated by repeated compression of microbubbles. Langmuir 2019, 35, 4238-4245.

[38] Zhang, J. F.; Fang, F.; Liu, B.; Tan, J. H.; Chen, W. C.; Zhu, Z. L.; Yuan, Y.; Wan, Y. P.; Cui, X.; Li, S. L. et al. Intrinsically cancermitochondria-targeted thermally activated delayed fluorescence nanoparticles for two-photon-activated fluorescence imaging and photodynamic therapy. ACS Appl. Mater. Interfaces 2019, 11, 41051-41061.

[39] Wang, S. H.; Shang, L.; Li, L. L.; Yu, Y. J.; Chi, C. W.; Wang, K.; Zhang, J.; Shi, R.; Shen, H. Y.; Waterhouse, G. I. N. et al. Metalorganic-framework-derived mesoporous carbon nanospheres containing porphyrin-like metal centers for conformal phototherapy. Adv. Mater. 2016, 28, 8379-8387.

[40] Yasui, K.; Tuziuti, T.; Kanematsu, W. Mysteries of bulk nanobubbles (ultrafine bubbles); stability and radical formation. Ultrason. Sonochem. 2018, 48, 259-266. 